\title{
Management of Complications after Major Gastrointestinal Surgery
}

\author{
Elke Muhl \\ Operative Intensivmedizin IOI-II/37a, Department of Anesthesiology and Intensive Care, University Medical Center Schleswig-Holstein, \\ Campus Lübeck, Lübeck, Germany
}

Leakage of esophagojejunal and esophagogastral anastomosis or bile leakage after pancreatic surgery are major complications. They are associated with a high mortality rate and a possibly long stay in the intensive care unit (ICU) due to organ dysfunction or organ failure after severe sepsis and septic shock, peritonitis, mediastinitis, or pleural empyema. The management of these complications mostly requires a multidisciplinary approach. Besides surgery, gastroenterology, radiology, and intensive care may be involved in the decision making regarding the treatment of these severe complications.

The management of the individual patient does not only depend on the location and size of the anastomotic leakage but also on its drainage, the reason of the leakage (i.e. extended necrosis), the risk profile of the patient, the risk of therapeutic options, the severity of illness, and last but not least the availability and expertise of specialists.

Most of the patients with the aforementioned complications require intensive care because of severe sepsis and septic shock. Technical success in the treatment of anastomotic leakages is an important aspect but is not sufficient in terms of the patient's survival. Volume management and catecholamine therapy controlled by hemodynamic monitoring, antibiotic therapy, and treatment of organ failure or insufficiency by e.g. renal replacement therapy and artificial ventilation are also important concerning further complications and the outcome of the patient. The intensive care specialist should be aware of clinical signs and laboratory values preceding such complications. Furthermore, the ICU staff should have some experience with postoperative complications and their treatment options.

Unfortunately, we do not have any controlled randomized multicenter studies about the outcome of therapies. Thus, decision making regarding the treatment of these patients with severe complications will be predominantly based on more or less large case control studies and expert opinions from different medical specialties.

This special issue of Visceral Medicine contains contributions of experts from gastroenterology, surgery, and radiology with a profound knowledge of the interdisciplinary management of these severe postoperative complications [1-6]. Each of the medical specialties has its limitations and very special indications regarding the treatment of anastomotic leakages. The process of decision making in the individual case primarily is an interdisciplinary process involving surgeon, endoscopist, radiologist, and intensive care specialist.

Kähler [1] reports on a variety of endoscopic methods for the treatment of gastroesophageal anastomotic leakages: fibrin glue, clipping, stenting, and endoscopic vacuum-assisted closure system. Providing a correct indication is crucial, and technical success of esophageal stenting in the management of esophageal anastomotic leaks and benign esophageal perforations can be expected in more than $90 \%$, while clinical success rates reach up to $81 \%$ [7].

The surgical treatment of leakages of hepaticojejunal anastomosis - a rare event - is addressed by Wellner and Keck [4]. In addition, the authors provide impressive pictures of the operation technique for repairing a hepato-intestinal anastomosis in the case of early leaks.

Especially in anastomotic problems occurring late after the operation, reoperation may be a relevant trauma for the septic patient, especially in the case of severe interenteric adhesions. Therefore, surgical intervention due to anastomotic leakages after gastrectomy and esophagectomy is mainly performed in critically ill patients and after the failure of conservative or endoscopic treatment. In this regard, leakage after upper gastrointestinal surgery is focused on by Hummel and Bausch [5].

Furthermore, the surgical treatment options of bile duct injury after cholecystectomy are given by Renz, Bösch and Angele [2]. The diagnosis and treatment of a bile duct injury after cholecystectomy is the domain of endoscopy. Reoperation is required for the definitive repair of complete dissection or complete obstruction of the bile duct [2]. The special risks associated with laparoscopic bile duct injuries as well as the classification of bile leakages and bile duct obstruction and the principles of surgical management are described in this article [2].

\section{KARGER}

() 2017 S. Karger GmbH, Freiburg

Fax +497614520714
Prof. Dr. med. Elke Muhl

Operative Intensivmedizin IOI-II/37a

Klinik für Anästhesiologie und Intensivmedizin

Universitätsklinikum Schleswig-Holstein, Campus Lübeck

Ratzeburger Allee 160, 23528 Lübeck, Germany

Elke.Muhl@uksh.de 
Percutaneous transhepatic biliary drainage may provide an internal drainage of hepaticojejunal anastomoses and is sometimes placed intraoperatively by the surgeon as a prophylaxis of anastomotic leakage. May and Hunold [3] report on rarely performed radiological interventions such as percutaneous transhepatic biliary drainage or stent in the case of anastomotic leakages of hepaticojejunal anastomoses.
Last but not least, the 'Interdisciplinary Discussion' of this special issue chaired by Muhl [6] extensively addresses further aspects of complications after major gastrointestinal surgery, e.g. septic shock, the importance of specialized centers, and antibiotic chemotherapy.

\section{References}

1 Kähler G: Anastomotic leakage after upper gastrointestinal surgery: endoscopic treatment. Visc Med 2017;33: DOI: $10.1159 / 000475783$.

2 Renz BW, Bösch F, Angele MK: Bile Duct injury after cholecystectomy: surgical therapy. Visc Med 2017;33: DOI: 10.1159/000471818.

3 May K, Hunold P: Leakage of hepaticojejunal anastomosis: radiological interventional therapy. Visc Med 2017;33:DOI: 10.1159/000469476.
4 Wellner UF, Keck T: Leakage of hepaticojejunal anastomosis: reoperation. Visc Med 2017;33:DOI: 10.1159/ 000471909.

5 Hummel R, Bausch D: Anastomotic leakage after upper gastrointestinal surgery: surgical treatment. Visc Med 2017;33:DOI: 10.1159/000470884

6 Muhl E (Chair): Management of complications after major gastrointestinal surgery (Interdisciplinary Discussion). Visc Med 2017;33:DOI: 10.1159/000475540.
7 Dasari BV, Neely D, Kennedy A, Spence G, Rice P, Mackle E, Epanomeritakis E. The role of esophageal stents in the management of esophageal anastomotic leaks and benign esophageal perforations. Ann Surg 2014;259:852-860. 